\title{
Low Power Energy Harvesting \& Supercapacitor Storage
}

\author{
Manish Anand ${ }^{1}$, A. M. Nagaraj ${ }^{2}$ \\ ${ }^{I}$ (M Tech Student, Department of EEE, Dayananda Sagar College of Engineering/VTU, India) \\ ${ }^{2}$ (Associate Professor, Department of EEE, Dayananda Sagar College of Engineering/VTU, India)
}

\begin{abstract}
This paper presents a hand cranked generator or an exercise bicycle to generate small amount of energy which is effectively stored in a supercapacitor Bank. Supercapacitors can absorb and deliver huge bursts of power in a short span of time owing to its low ESR (Equivalent Series Resistance).If more charging current is given to the battery it may lead to excessive heating and decreases the life of the battery but the charging current limit of supercapacitors is very high. Thus it can be charged rapidly which is a very useful feature for energy harvesting applications. The energy generated by hand cranking or coupling the generator with an exercise bicycle rapidly charges the supercapacitor bank via a current limiter. The supercapacitor Bank can then be effectively discharged using a low voltage boost converter to store the energy in a battery or to power small devices. This way the energy generated by the exercise bicycle which is an ambient source of energy can be effectively utilized which would have otherwise gone wasted.
\end{abstract}

Keywords - Energy Harvesting; energy storage, charge balancing, supercapacitors, DC-DC converters.

\section{INTRODUCTION}

Powering small electronic gadgets in remote area can be a challenging task. In some villages which are far away from electric grid people still use kerosene for lighting their lamps. Instead some form of energy harvesting device can be used to produce small amount of power which will be sufficient to power small devices like radio, LED lamps \& cell phone charging.

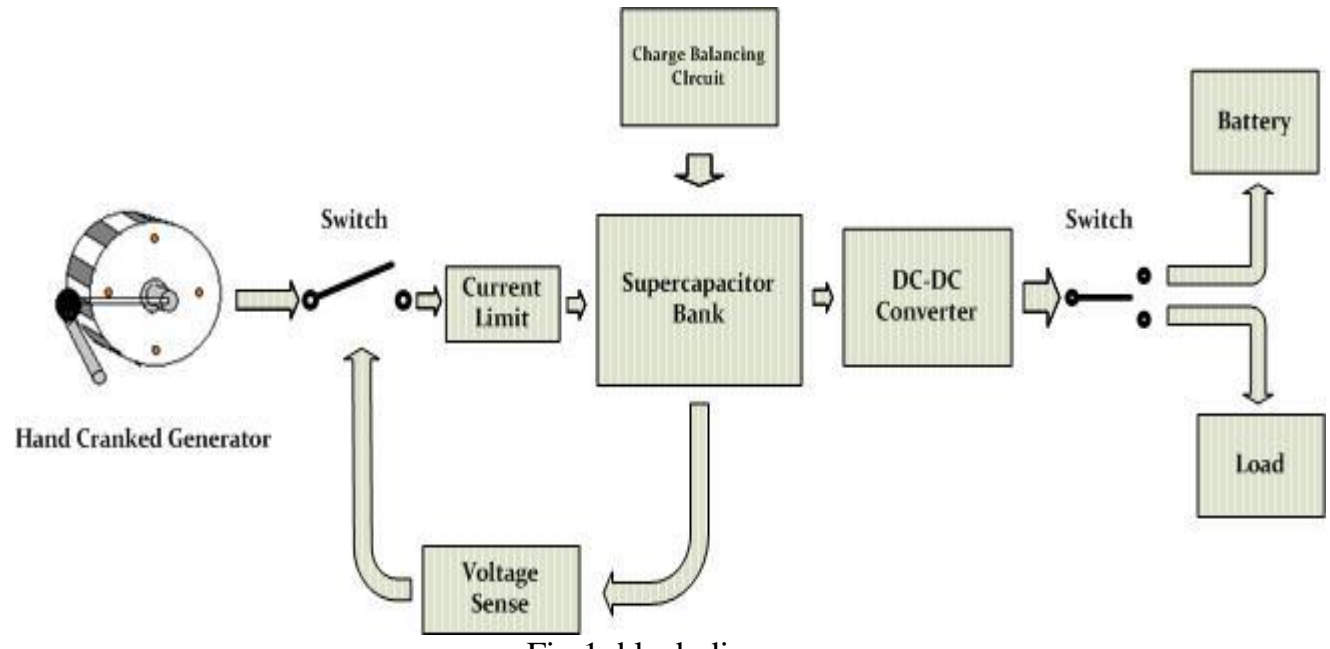

Fig.1. block diagram

Energy harvesting devices capture excess energy from everyday movements. They capture short burst of intermittent flow of energy [1]. This project aims to make an energy harvesting arrangement that can be cranked for few minutes to generate enough energy to power small devices. Exercise bicycle can be used as an ambient source of energy [3]. It can easily generate 20-30W of power if proper arrangement is made. Some sort of storage device is needed which absorbs the energy generated in short span of time which can be utilized gradually in a relatively longer time span. So some energy storage device is needed which quickly absorbs the intermittent flow of energy. Thus supercapacitors are being used as energy storage device owing to its quick energy absorbing capability which is an essential requirement in energy harvesting device. A supercapacitor is a capacitor which can store large quantity of charge and has capacitance in order of thousands of Farads. Supercapacitor has a typical time constant of approximately one second. One time constant reflects the time necessary to charge a capacitor $63.2 \%$ of full charge or discharge to $36.8 \%$ of full charge.

When the supercapacitor bank discharges while utilizing the stored energy its voltage drops. Most of the loads work at constant voltage. So a DC-DC converter is required to give a stable voltage output to power loads i.e. LEDs, cellphone charging etc. 
Care has to be taken regarding the supercapacitor charging current. Some form of arrangement is needed to regulate the charging current flow as the supercapacitor Bank behaves at dead short at uncharged state.

The energy stored in the supercapacitor Bank has to be utilized soon after storing it due to higher leakage current of supercapacitor than the battery. So we must generate the energy just before using it. Otherwise it can be transferred to the battery if we want to store it for longer time.

When connecting the supercapacitors in series a charge balancing circuit is used to evenly distribute the charge across the series connected capacitors and save them from overvoltage condition which might deteriorate their lifetime.

Supercapacitor as energy storage medium is an emerging field. Research is going on to increase its Energy Density and reduce its leakage current. With the prices of supercapacitor decreasing in coming years due to popularity and improvement in technology, they can even replace batteries in certain applications.

\section{ENERGY HARVESTING SETUP}

Initially a small model using $3 \mathrm{~W}$ DC generator has been made. Later this setup can be scaled up using bicycle power generator which will generate 20 to $30 \mathrm{~W}$ power. The energy harvesting \& supercapacitor storage setup used is very versatile. Although a hand cranked generator is being used as the energy generating device, the same setup can be used for recovering regenerative braking energy from electric vehicle. These are the following components as shown in Fig.1:

- Hand Cranked Generator

- Current Limiter

- Voltage Sense Circuit

- Supercapacitor Bank

- Charge Balancing Circuit

- DC-DC Convertor

- Load

\subsection{Hand Cranked Generator}

A small PMDC motor is used a hand cranked generator using proper gear or pulley arrangement to rotate it at higher speed even when the cranking speed is low. PMDC generator is a very good choice for energy harvesting applications as it does not require field supply [4]. A 12V DC generator with power output of 2-3W is used. Voltage Vs. speed graph helps to predict the input voltage range going to the DC-DC converter.

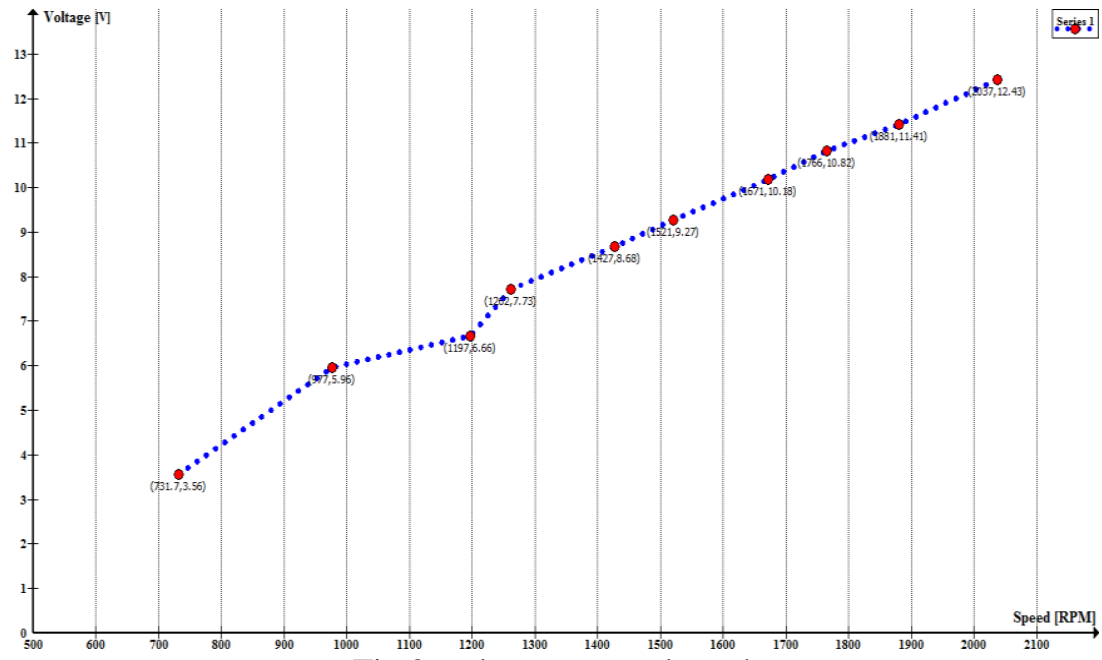

Fig.2 voltage vs speed graph

\subsection{Current Limiter}

Supercapacitor bank will behave as a short circuit to the hand cranked generator when in uncharged or low charge state. So a current limiting arrangement is required which will offer some resistance to the current flow. Here LM317 based current limiter has been used. In this particular configuration the current flow is limited to $250 \mathrm{~mA}$. The current flow can be programmed from $0-1.25 \mathrm{~A}$ using suitable resistor value.

: Iout $=1.25 / \mathrm{R} 1$

Since some work is done to rotate the hand cranked generator, if the current draw is higher for the Supercapacitor Bank charging, more work has to be done i.e. more torque (Torque=Force*Distance) has to 
applied to rotate the hand cranked generator .So by adjusting the current flow in the supercapacitor bank we can adjust the torque needed to rotate the hand cranked generator.

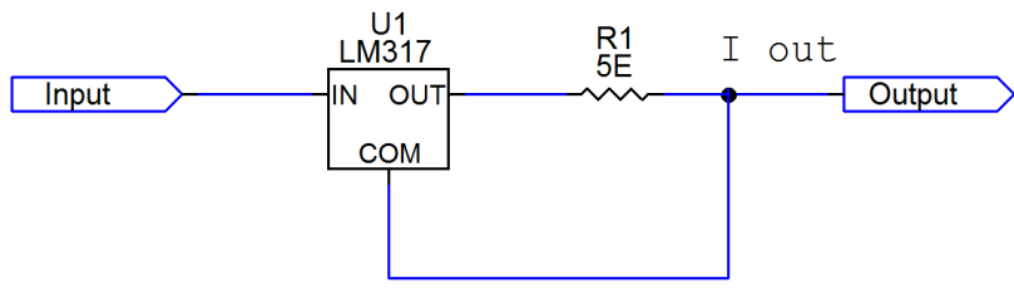

Fig.3. current limiter

\subsection{Voltage Sense Circuit}

It disconnects the supercapacitor bank from the hand cranked generator when it is charged to set voltage and prevents overcharging. It has been made using single supply dual comparator LM 393. One comparator disconnects the charging when the supercapacitor bank voltage reaches a preset level programmed by the preset R6. Other comparator is used to give the supercapacitor bank voltage indication.

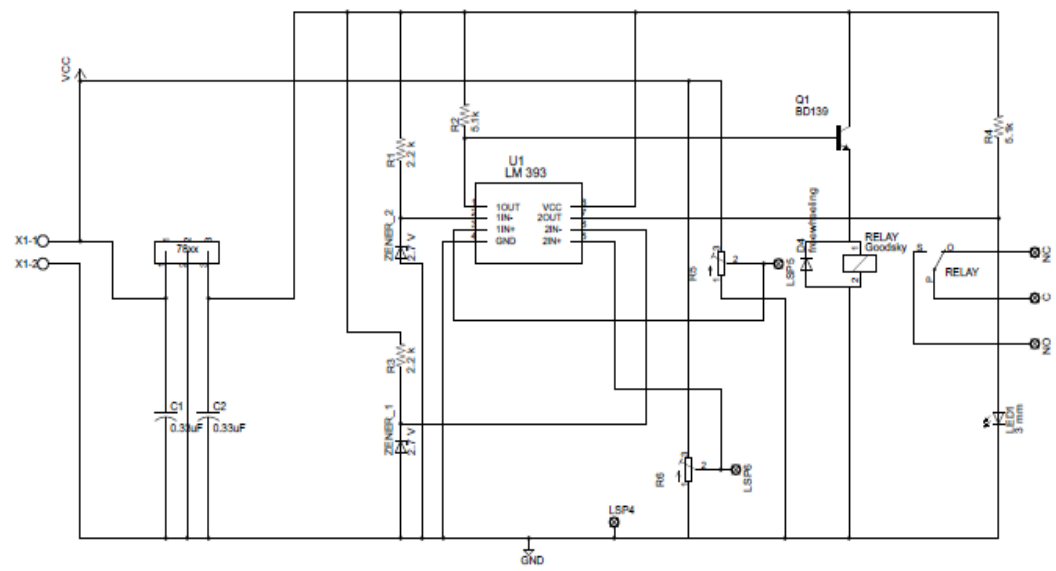

Fig.4 voltage sense circuit

\subsection{Supercapacitor Bank}

Supercapacitor Bank is made by connecting supercapacitors in series. Each individual supercapacitor is rated for $2.7 \mathrm{~V}$. So for increasing the voltage rating they need to be connected in series. Sizing of supercapacitor Bank is an important aspect. It depends on how much energy need to be stored. Three 100F supercapacitors in series have been connected to make $33.33 \mathrm{~F} / 8 \mathrm{~V}$ supercapacitor Bank which will store energy given by $: \mathrm{E}=1 / 2 \mathrm{C} \mathrm{V}^{2}=1 / 2[33.33][7.5]^{2}=937.4$ Joules

\subsection{Charge Balancing Circuit}

A charge balancing circuit is needed to avoid any single capacitor in the supercapacitor Bank to be overcharged [2]. While charging slight voltage difference will be there between the individual supercapacitors due to slightly different parameters such as internal resistance even for same part number, when they are connected in series. An active charge balancing circuit has been used. It consists of a comparator controlled switch which bypasses small amount of current when one supercapacitor is slight more charged than the other to equalise the charge distribution.

\subsection{DC-DC Converter}

The supercapacitor bank voltage will drop gradually as it will discharge but a constant output voltage is required for powering the loads. So a buck converter based on MC34063 IC is used. The buck converter has been designed using the design formulae given in the datasheet.

The utilization of power from the supercapacitor Bank depends on the input voltage range of the DCDC converter. Since the energy in the capacitor is proportional to the voltage squared according to $: \mathrm{E}=1 / 2 \mathrm{C} \mathrm{V}^{2}$

It is possible to utilize approximately $75 \%$ of the available energy if the application utilizes from the rated voltage to $1 / 2$ rated voltage of the capacitor. 


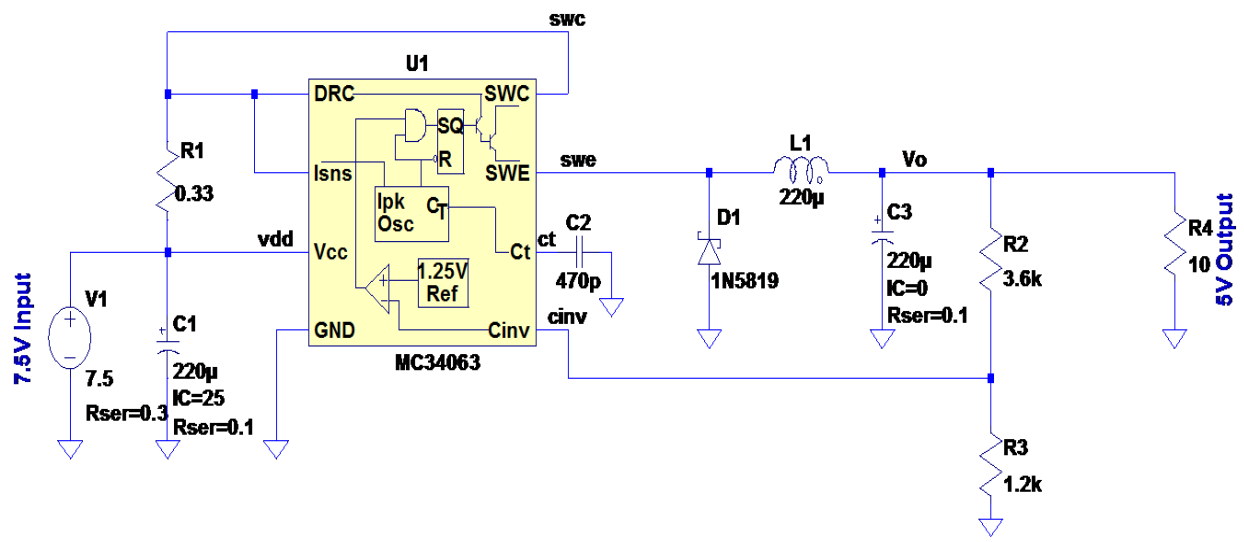

Fig.5 buck converter

Low ESR electrolytic capacitors C1 \& $\mathrm{C} 3$ have been used to minimize the losses in the DC-DC converter. R1 is used for current limit in case of short circuit at the load side. The output voltage can be varied by altering the arrangement of voltage sense potential divider made by R2 \& R3.

Some other ASICs (Application Specific ICs) like LM2576, LM2596, and LM2577 also can be used to make DC-DC converters with added features like soft start, overcurrent protection, shutdown etc. efficiency.

Linear Technology offers a lot of alternatives for energy harvesting applications with very high

PCB designing for the DC-DC converters is done using EAGLE PCB design software. Proper layout technique is used like keeping the trace of freewheeling diode, input and output capacitors as short as possible. All the high current path traces are made thicker than the rest.

\subsection{Load}

LED load is connected to DC-DC converter. Dimmer circuit is developed using TLC555 IC which is a CMOS version of 555 Timer and can achieve very high frequencies till 1.2 MHz. This ensures flicker free dimming of white LEDs. For higher frequency applications $>50 \mathrm{kHz}$, TIP31C can be replaced with a suitable rating MOSFET and a MOSFET driver.

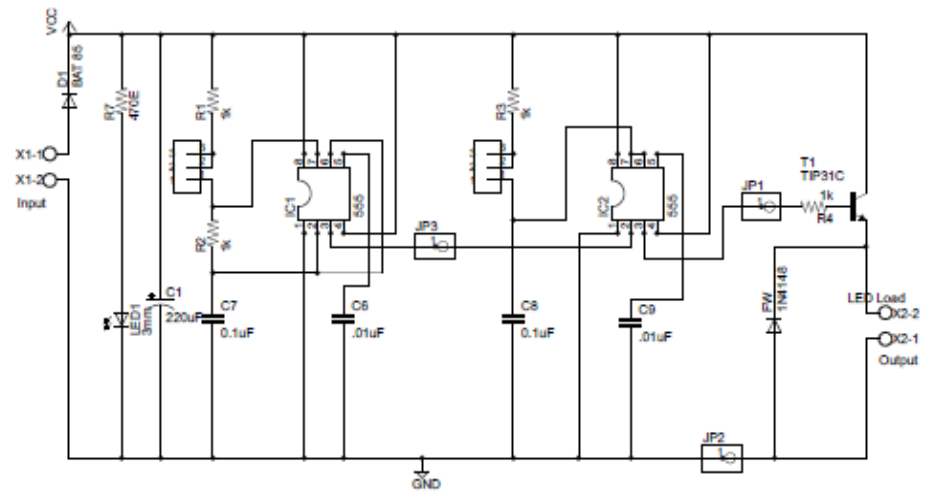

Fig.6 LED PWM brightness controller

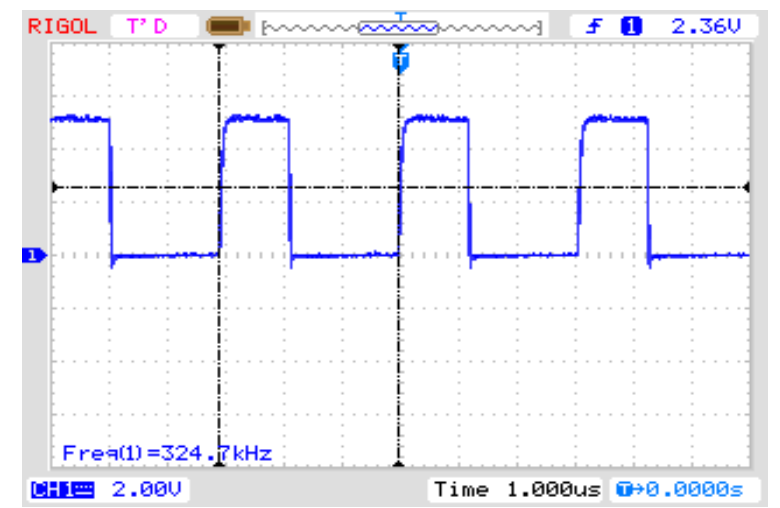

Fig.7 PWM waveform from the LED brightness controller 
Another load is cell-phone whose charging requires the output voltage of the buck converter to be set at $5 \mathrm{~V}$. There are other hand cranked chargers available in the market but the advantage of this arrangement is that it will charge the cell-phone even for some time after the cranking stops because of the stored energy in the supercapacitors. The previously available chargers only charge during the cranking time with lesser efficiency.

\section{Simulation}

Simulation of Buck Converter using MC34063 Switching Regulator IC has been done in LT Spice. LT Spice is free Spice simulation software from Linear Technology which was previously called switcher CAD. It provides schematic capture and waveform viewer. Component library mostly contains Linear Technology but even a 3rd party model can be imported. LT Spice IV netlist can be imported into any PCB design software.

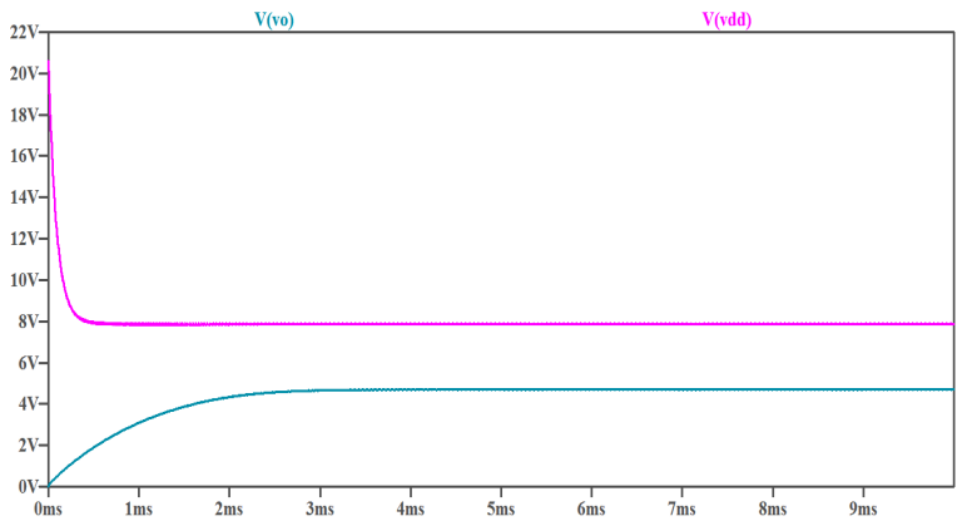

Fig.8 input and output voltage

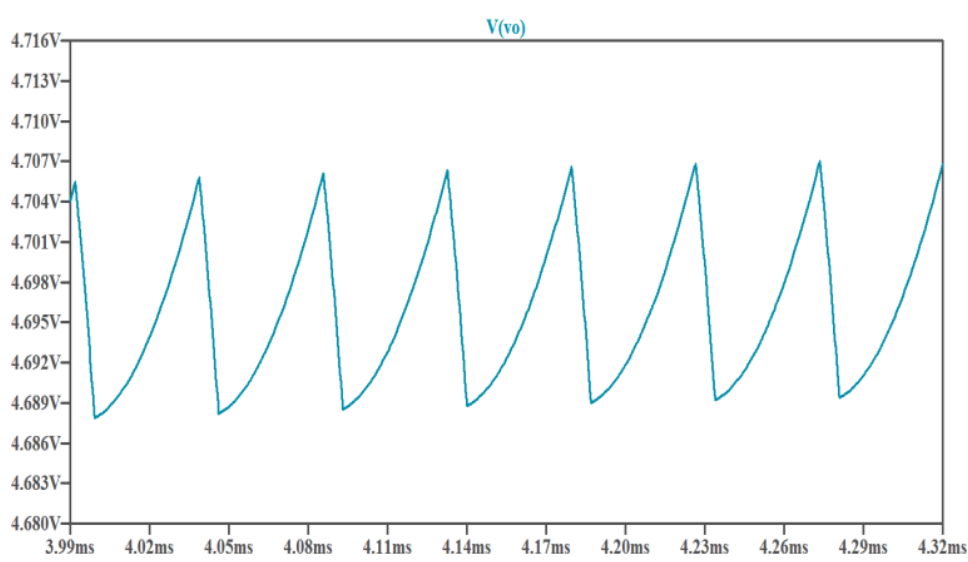

Fig.9 output voltage ripple

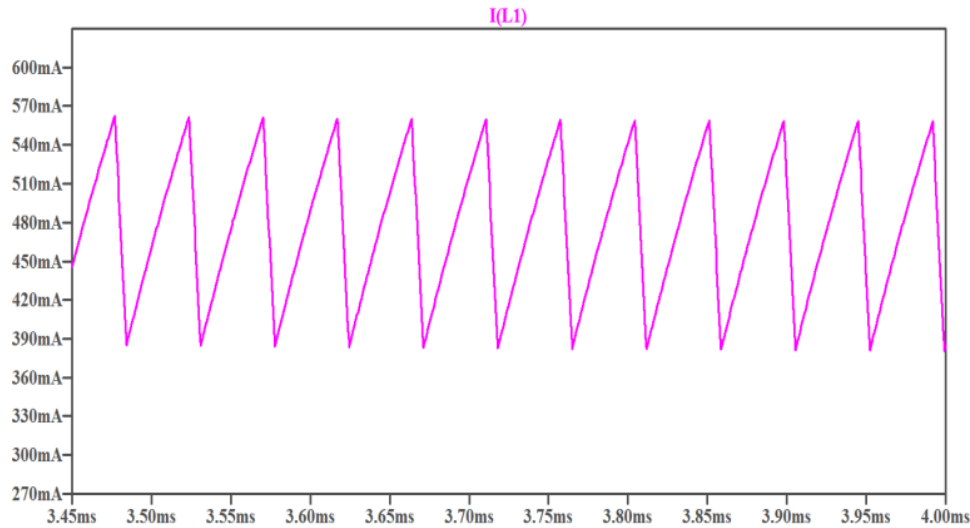

Fig.10 inductor current ripple

The output voltage is $5 \mathrm{~V}$ as expected with an input voltage range of $6-12 \mathrm{~V}$. The output voltage ripple is $100 \mathrm{mV}$. The current ripple in the inductor is $700 \mathrm{~mA}$. The simulation gives an idea of the voltage range of the supercapacitor bank which can be utilised by the DC-DC converter for powering any load at steady voltage. 


\section{CONCLUSION}

The paper demonstrates an arrangement for low power energy harvesting and storage device for powering small electronic gadgets. The energy absorbing ability of this arrangement is much better than battery based energy storage systems in energy harvesting. The arrangement can be used for off grid applications in remote areas. This energy harvesting arrangement does not depend on any intermittent sources like wind or sunlight. The energy stored must be utilized soon due to high leakage current of supercapacitors. If energy has to be stored for longer duration then it must be transferred to a battery using a DC-DC converter. Most electronics have a minimum voltage threshold for utilization, limiting the effective utilization voltage of the supercapacitor although there is no limitation in the supercapacitor itself. So, better utilization of the stored energy can be done by using low voltage DC-DC converters. Future work includes developing regenerative brakes for bicycle for urban use where frequent braking is required. So, whenever brakes are applied on a busy road the energy is recovered back in the supercapacitor bank instead of getting wasted in the brakes.

\section{Acknowledgements}

The authors thank Dayananda Sagar College of Engineering, Bangalore for the infrastructure and support. A special thanks to Dr. K. Shanmukha Sundar, HOD, EEE Department, Dayananda Sagar College of Engineering, Bangalore for mentoring and encouraging us to do this project. Also special thanks to the staff at the college for their continuous support.

\section{Proceedings Papers:}

\section{REFERENCES}

[1] Yildiz, F. Low Power Ambient Energy Harvesting, Conversion, and Storage Circuits, Information Technology: New Generations, 2009. ITNG '09. Sixth International Conference on 27-29 April 2009.

[2] Dirk Linzen, Stephan Buller, Eckhard Karden and Rik W. De Doncker, Analysis and Evaluation of Charge-Balancing Circuits on Performance, Reliability, and Lifetime of Supercapacitor Systems: IEEE Transactions on Industry Applications, Vol. 41, September/October, NO. 5, 2005.

Journal Papers:

[3] Joseph A. Paradiso, Thad Starner, Energy Scavenging for Mobile and Wireless, IEEE Pervasive Computing archive, Volume 4 Issue 1, January 2005.

[4] Yildiz, F., Potential Ambient Energy-Harvesting Sources and Techniques, The Journal of Technology Studies. 